\title{
Development and Optimization of an LC-MS/MS Method for Dosage Form of Ergocalciferol (Vitamin $\mathrm{D}_{2}$ ) in Human Plasma
}

\author{
Srinivas $\mathrm{A}^{* 1}$ and Tamilselvi $\mathrm{M}^{2}$ \\ ${ }^{1}$ Department of Genetics, Osmania University, Hyderabad, 500 007, Telangana, India \\ ${ }^{2}$ Value Added Corporate Services Pvt Ltd, Besant Nagar, Chennai, 600090, India
}

${ }^{*}$ Corresponding author: Srinivas A, Department of Genetics, Osmania University, Hyderabad 500 007, Telangana, India, E-mail: srinivasmessage@gmail.com

Citation: Srinivas A, Tamilselvi M (2017) Development and Optimization of an LC-MS/MS Method for Dosage Form of Ergocalciferol (Vitamin $\mathrm{D}_{2}$ ) in Human Plasma. J Bioeq Stud 3(1): 102

Received Date: Feb 24, 2017 Accepted Date: June 26, 2017 Published Date: July 27, 2017

\begin{abstract}
Accurate measurement of ergocalciferol in biological samples has become easier using high end mass spectra. Currently, various LCMS/MS methods have been developed to quantify ergocalciferol. Use of LC-MS/MS has accomplished to develop accurate method that could quantify ergocalciferol in large number of samples where it should be analysed in the blood immediately after dosage. The objective of this study is to optimize chromatographic conditions and evaluate the LC-MS/MS method for accurate quantification of dosage form of ergocalciferol in human plasma. Extraction and separation of ergocalciferol in human plasma was achieved using methanol liquid-liquid extraction and by a reverse phase $\mathrm{C}_{8}$ column, respectively. The residue after liquid-liquid extraction was dissolved using PTAD reagent along with the reconstituted solution to detect and quantify ergocalciferol by LC-MS/MS. Detection was performed on a quadrupole tandem mass spectrometer (LC-MS/MS) equipped with atmospheric pressure chemical ionization (APCI) source. The method was validated according to FDA guidelines and certified reference material was used. The LOQ for analyte tested was $5.44 \mathrm{ng} / \mathrm{mL}$, in medium-MQC it was $65.123 \mathrm{ng} / \mathrm{mL}$ and in high-HQC it was $120.598 \mathrm{ng} / \mathrm{mL}$ and the quality control samples did not exceed 20 and 15\% CV, respectively. Accuracy of the method for determination of ergocalciferol was validated and found to be in the range of $95.40-103.09 \%$. This method can be used for quantification of ergocalciferol present in the systemic circulation via external consumption in the form of tablets.
\end{abstract}

Keywords: Ergocalciferol; Precipitation; LLE; APCI; PTAD; LC-MS/MS

\section{Introduction}

Ergocalciferol (Vitamin $\mathrm{D}_{2}$ ), is a fat-soluble vitamin that helps body to absorb calcium and phosphorus. Proper quantity of vitamin $\mathrm{D}_{2}$, calcium, and phosphorus are important for having healthy strong bones. Vitamin $\mathrm{D}_{2}$ is synthesized by the body when skin is exposed to sunlight. Nominal level of vitamin D in body is considered to give a beneficial effect on decreasing the risk of diabetes, obesity, wheezing, multiple sclerosis, allergy, asthma, mental disease, depression, schizophrenia, kidney problems and hypertension. Several studies were done on prenatal and postnatal vitamin D status during the pregnancy, infancy and early childhood and its deficiency is linked with lower adult bone mass and increased fracture risk in life [1]. In addition, a number of research studies demonstrated the role of vitamin D in blood pressure regulation, autoimmunity, regulation of cell growth and metabolic diseases [2-8].

Many recent reports have assessed prevalence of vitamin D deficiency around the world. A number of clinical trials and epidemiological studies have proved that vitamin $\mathrm{D}_{2}$ has a role in prevention of many chronic diseases and its deficiency is associated with an increased risk of diseases like osteoporosis, autoimmune disorders, diabetes, hypertension, autism, infections and various cancers. Sunscreen, protective clothing, limited sunlight exposure, dark skin, and ageing prevent the body from having sufficient vitamin D synthesized from the sunlight. Ergosterol is commercially produced by treating ultraviolet irradiation of provitamin D sterol, which exists in plants. Vitamin $\mathrm{D}_{2}$ deficiency in body elevates serum levels of parathyroid hormone (PTH) and leads to high bone resorption and in turn cause osteoporosis. Severe vitamin D deficiency causes rickets or osteomalacia [9]. Vitamin $\mathrm{D}_{2}$ with calcium is used to treat or prevent bone loss (osteoporosis). Vitamin $\mathrm{D}_{2}$ is also used to treat and prevent bone disorders (such as rickets, osteomalacia and cardiovascular diseases). 
Several studies were published recently to quantify vitamin $\mathrm{D}$ and its metabolites in biological matrices. The levels of 25-hydroxyvitamin D (25-OH-D) in blood are the indicator of vitamin D status. The biologically active form of vitamin D, is produced when it is hydroxylated (25-OH-D) and is usually present in the plasma in low pg/ml ranges. Two major forms of vitamin $\mathrm{D}$ can be found in the body. These include vitamin $\mathrm{D}_{3}$, which is endogenously produced, and vitamin $\mathrm{D}_{2}$, which is only present when it is given in food or as a supplement [11]. Vitamin $\mathrm{D}_{2}$ status is largely indicated by 25 -hydroxyvitamin $25(\mathrm{OH}) \mathrm{D}_{2}$ levels in serum since it is the main storage metabolite of vitamin $\mathrm{D}_{2}$ representing its accumulation both from diet and cutaneous synthesis. It is present in low concentrations in both foods and biological tissues. Vitamin $\mathrm{D}_{2}$ and $\mathrm{D}_{3}$ both are clearly not equivalent. Studies suggest differential response of vitamin $\mathrm{D}_{2}$ and $\mathrm{D}_{3}$ in humans [3]. Vitamin $\mathrm{D}_{2}$ is not an appropriate nutrient for supplementation or fortification of foods [3]. In tropical country like India 30 to $50 \%$ of children and adults had 25 -hydroxy vitamin $\mathrm{D}_{2}$ levels under 20 ng per milliliter [6].

An increased understanding of the importance of vitamin $\mathrm{D}_{2}$ has led to a growing interest in the analytical methods used in research for the detection of vitamin $\mathrm{D}_{2}$ and its metabolites. Derivatization method is used for increasing the sensitivity of LC-MS/ MS to detect the given analyte [12]. PTAD (4- phenyl-1,2,4-triazoline-3,5-dione), Amplifex ${ }^{\mathrm{m}}$ diene and PyrNO reagent are used in derivatization process to react with vitamin Ds (including vitamin $\mathrm{D}_{3}$, vitamin $\mathrm{D}_{2}$ and their respective metabolites) within minutes and unreacted reagents elute very early in reversed phase LC and enhance sensitivity and lower the detection limits [13-18]. Most of the recently studied methods states that use of PTAD reagent improves sensitivity in LC-MS/MS analysis. PTAD derivatization reaction takes place on specific sites such as the s-cis-diene structure in vitamin D related compounds or functional groups that include hydroxyl or carbonyl groups in steroids and oxysterols of the compound. Therefore, it is essential to quantify vitamin $\mathrm{D}_{2}$ in human plasma using PTAD derivatization. Sample preparation techniques used for vitamin $\mathrm{D}$ metabolites determination include LLE [7], SPE in serum and saponification [8]. Extraction method from serum is based on acetonitrile and a short $\mathrm{C}_{18}$ column to separate ergocalciferol from other metabolites. Studies found APCI to be a suitable ionization source for quantification of ergocalciferol [19].

Determination of vitamin $\mathrm{D}$ is required to detect for its deficiency, but also increasingly to adjust therapeutic targets and monitor safety and efficacy of treatment. The difficult task in ergocalciferol analysis is to establish the ionization efficiencies for low levels of specific analytes expected in human plasma samples. The claim of the recently published method states that high sensitivity ( 0.10 $\mathrm{ng} / \mathrm{mL})$, low imprecision, low sample volume $(100 \mu \mathrm{L})$ and data processing done by Watson LIMS software provide superior data integrity during method validation and study sample analysis successfully applied to a bioequivalence study of $1.25 \mathrm{mg}$ ergocalciferol capsules in 12 healthy subjects [20]. In a similar line simultaneous assay of vitamin D metabolites [21] and circulating concentrations of vitamin D metabolites in serum [22] studies were done. Accordingly, developing a standard method for quantification is crucial to help and improve effective formulation of ergocalciferol but also increasingly to adjust therapeutic targets and monitor safety and efficacy of treatment. Hence, the purpose of present study is to develop and validate method for determination of ergocalciferol for the dosage form of ergoalciferol in human plasma.

\section{Materials and Methods}

\section{Chemicals and reagents}

Analytical standards for $\mathrm{D}_{2}, \mathrm{D}_{3}$, as well as pooled plasma, $\mathrm{D}_{3}$ and ergocalciferol were purchased from Clearsynth Private Limited and erogocalciferol was procured from US Pharmacopea. Human plasma was obtained from VHS, Chennai, acetonitrile ( $\geq 99.8 \%)$, methanol ( $\geq 99.9 \%)$, isopropanol $(\geq 99.5 \%)$, and hexane $(\geq 99.5 \%)$ of HPLC grade were used. Deionized water was produced by a Milli-Q gradient system from Millipore (Bedford, MA).

\section{Stock and working standard solutions}

Stock solutions of ergocalciferol in the concentrations of $90.929 \mathrm{ng} / \mathrm{mL}$ to $7583.283 \mathrm{ng} / \mathrm{mL}$ were prepared by serially diluting the intermediate solution and were stored at $2-8{ }^{\circ} \mathrm{C}$. Internal standard (IS) solution of $1 \mathrm{mg} / \mathrm{ml}$ was prepared by using $\mathrm{D}_{3}$ in acetonitrile solution, from the stock solution.

\section{Preparation of calibration curves, quality control samples}

Calibration standards were prepared at a concentrations range of 1.819 to $151.666 \mathrm{ng} / \mathrm{mL}$ by adding $10 \mu \mathrm{L}$ of individual calibration working solutions with $180 \mu \mathrm{L}$ of PBSA solution (10 mM phosphate buffer, $\mathrm{pH} 7.5 ; 140 \mathrm{mM} \mathrm{NaCl} ; 8 \% \mathrm{BSA})$ and $10 \mu \mathrm{L}$ of IS (20 ng/ $\mathrm{mL}$ final concentration). Quality control (QC) samples were prepared independently from the QC stock solution in a similar way of calibration standards at concentrations of $5.44 \mathrm{ng} / \mathrm{mL}$ (LQC), $65.123 \mathrm{ng} / \mathrm{mL}$ (MQC) and $120.598 \mathrm{ng} / \mathrm{mL}$ (HQC) for ergocalciferol. In addition, standard reference sample plus IS and PTAD reagent were mixed together along with spiking solution to obtain aqueous recovery QC.

\section{Sample Collection and Processing}

Blood samples were collected in vacutainer tubes. The samples were immediately transferred and kept in ice. Later the samples were centrifuged at $4000 \mathrm{rpm} / 4^{\circ} \mathrm{C}$ for $10 \mathrm{~min}$ and the separated plasma was collected and stored at $-70{ }^{\circ} \mathrm{C}$ until analysis. The samples were centrifuged within $1 \mathrm{hr}$ and $30 \mathrm{~min}$ from the time of sample collection to protect sample integrity. 
Ergocalciferol is extracted by using protein precipitation followed by liquid-liquid extraction method and then derivatized using PTAD reagent (4-Phenyl-1, 2, 4-triaazoline-3, 5-dione). To evaluate extraction efficacy, solvent of acetonitrile, acetone, methanol, isopropanol and hexane were studied. For each extraction, $200 \mu \mathrm{L}$ of sample was taken in the pre labeled RIA vial and added $50 \mu \mathrm{L}$ of IS $\mathrm{D}_{3}$ and vortexed well using cyclomixer, after which $0.5 \mathrm{~mL}$ of methanol was added and vortexed well. It was followed by 1.0 $\mathrm{ml}$ of hexane addition to the sample and vortexed at $2500 \mathrm{rpm}$ for 10 minutes and centrifuged at $4500 \mathrm{rpm}$ for $10 \mathrm{minutes}$ at $4{ }^{\circ} \mathrm{C}$. Supernatant of $0.6 \mathrm{ml}$ was transferred directly into RIA vial and evaporated using nitrogen pressure at $40{ }^{\circ} \mathrm{C}$ till it dried. $50 \mu \mathrm{L}$ of $0.5 \mathrm{mg} / \mathrm{ml}$ PTAD reagent was added to the residue along with $400 \mu \mathrm{L}$ of reconstitution solution. $10 \mu \mathrm{L}$ of sample was taken from the reconstitution solution in a vial and transferred to an auto sampler and analyzed by using LC-MS/MS.

\section{LC-MS/MS}

The LC-MS/MS analysis was performed by using a Thermo TSQ Ultra (MS/MS) thermo Finnegan surveyor LC using APCI positive ionization mode system consisting of a binary pump, degasser and auto sampler. Column Zorbax SBCN (4.6 mm75mm, $3.5 \mathrm{micron}$ ) was used for reverse phase separation. Solvent A was water and solvent B was methanol containing $10 \mathrm{mM}$ ammonium formate with $0.1 \%$ formic acid $(75: 25 \mathrm{v} / \mathrm{v})$. The mobile phase flow rate was $0.8 \mathrm{~mL} / \mathrm{min}$ and injection volume was $10 \mu \mathrm{L}$. Ergocalciferol $\left(\mathrm{D}_{2}\right)$ was detected in positive mode using multiple reactions monitoring (MRM) technique.

The source conditions were as follows: capillary temperature, $250{ }^{\circ} \mathrm{C}$; vaporizer temperature, $250{ }^{\circ} \mathrm{C}$; sheath gas pressure 50 psi; capillary voltage, $250 \mathrm{~V}$; The mass spectra was set up to allow the $[\mathrm{M}+\mathrm{H}]+$ ions of vitamin $\mathrm{D}$ to pass the first quadrupole (Q1) into the collision cell (Q2), fragmented and corresponding fragments were monitored through third quadrupole (Q3). A total of three transitions were monitored for analyte and corresponding IS.

\section{Method validation}

Calibration curves of ergocalciferol were obtained by plotting the peak-area ratios of analyte and IS versus nominal concentrations in the range of 1.819 to $151.666 \mathrm{ng} / \mathrm{mL}$. The linearity was determined using correlation coefficient $\left(\mathrm{R}^{2}\right)$ of the curves by means of weighted least-squares linear regression method and found to be greater than 0.99 .

Precision and accuracy were calculated using extracts of QC samples prepared in PBSA at concentrations of 1.821, 5.444, 65.123, and $120.598 \mathrm{ng} / \mathrm{mL}$ for LLOQ, LQC, MQC and HQC respectively. For intraday precision, samples were reanalyzed for three times on a single day and for inter-day precision once in three consecutive days, respectively. All the three batches were found to be within the acceptance criteria of $\% \mathrm{CV} \pm 20 \%$ at LLOQ level and $\pm 15 \%$ at LQC, MQC and HQC levels. Concentrations were calculated from calibration curves prepared as described above. Precision study indicates that the present method can be used for the quantitative estimation of ergocalciferol $\mathrm{D}_{2}$ in human plasma. In this method the unknown concentrations were determined from the area ratio between non-deuterated (unknown quantity in sample) and deuterated $\mathrm{D}_{3}$ (known quantity of IS) forms of corresponding analytes. The accuracy was calculated by the following equation: (mean of determined concentration/nominal concentration) $\times 100$, and expressed in percentage (\%). The precision was evaluated by the relative $\mathrm{CV}(\% \mathrm{CV})$ and the results obtained were within the range.

\section{Results}

\section{Optimization of MS/MS conditions}

The difficult task in ergocalciferol analysis is to establish the ionization efficiencies for low levels of specific analytes expected in human plasma samples. In general ESI is used in combination with LC-MS for analysis of several vitamins. However, the effectiveness of this method of ionization is analyte-dependent. The structure of ergocalciferol $\mathrm{D}_{2}$ molecule (Figure 1) suggests that it would not readily protonate during ESI. Thus, atmospheric pressure chemical ionization (APCI) has been used in combination with a MRM technique for sensitive detection of ergocalciferol and its metabolites in human plasma. Therefore, APCI-MS/MS mode is used for quantification of ergocalciferol $\mathrm{D}_{2}$. During method development, we have tried out various methods to standardize the sample extraction and preparation, in order to get a high precision and accuracy.

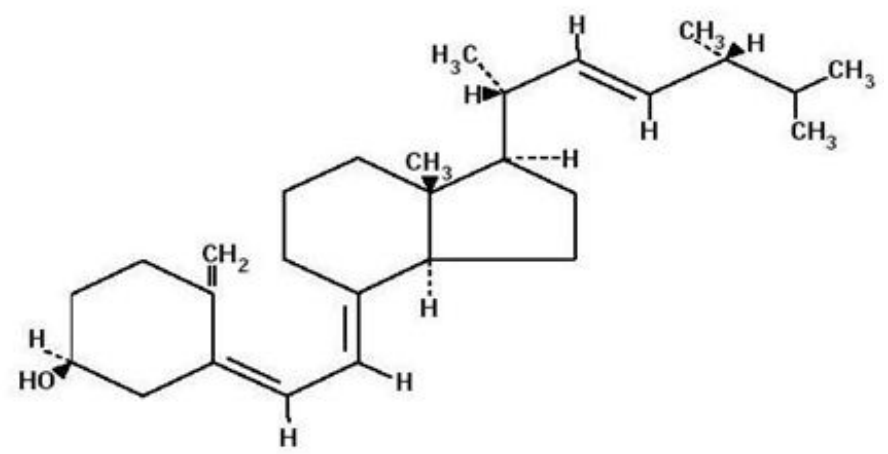

Figure 1: Chemical structure of ergocalciferol 


\section{Method Validation}

Optimized LC-MS/MS conditions were validated using purified standards. Method validation was done as per USFDA guidelines [23]. The LOQ and linearity of detector response was quantified using nine calibration standards ranging concentration from 1.819 to $15.666 \mathrm{ng} / \mathrm{mL}$ in a $10 \mu \mathrm{L}$-injection volume. A standard curve was generated by plotting calculated peak area ratios of individual vitamin D metabolites to the corresponding IS versus their nominal concentrations. The best fit of individual standard curves was achieved using a first-order linear regression within the tested ranges $\left(\mathrm{R}^{2} \geq 0.995\right)$ (Figure 2). Plasma concentrations were calculated by dividing the analyte peak area and the area of the corresponding internal standard vitamin $\mathrm{D}_{3}$. A weighted $1 / \mathrm{x}^{2}$ linear regression was performed to determine the concentration of the analyte. The lower limit of quantitation for ergocalciferol is $1.819 \mathrm{ng} / \mathrm{mL}$ and the upper limit of quantitation is $151.666 \mathrm{ng} / \mathrm{mL}$.

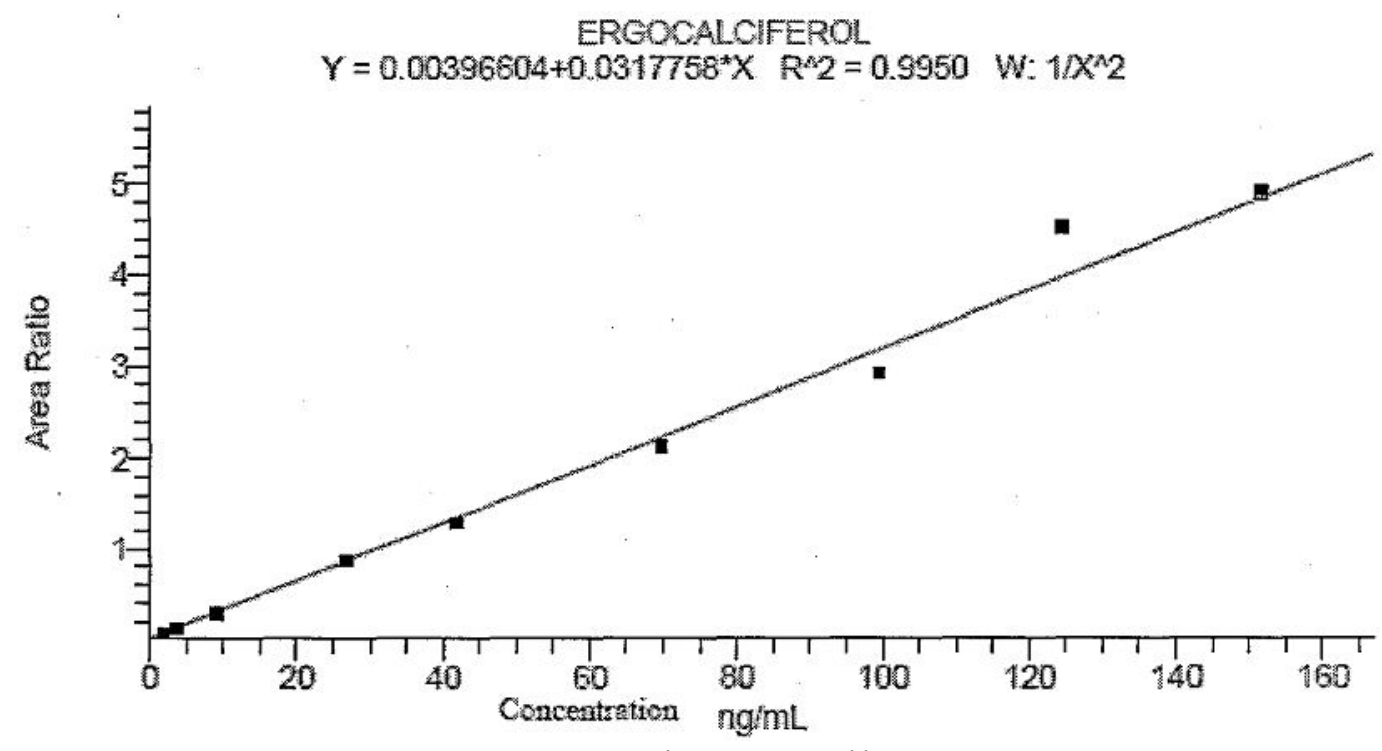

Figure 2: Representative chromatogram calibration curve

\section{Matrix Effect}

Six different lots of (including hemolytic and lipemic) blank human plasma were processed in duplicate and reconstituted with aqueous HQC and LQC samples. Extracted and aqueous samples were then compared to determine the matrix factor for analyte and IS. The results showed no obvious interference.

\section{Precision and accuracy of the analytical method}

The accuracy and precision of this method based on intra-day repeatability was performed by replicate injections ( $\mathrm{n}=6$ ) directly in plasma of three spiked solutions of low, medium and high concentration levels. The inter day precision of the method was established again in plasma using the same concentration range. A triplicate determination of each concentration was done over a period of three consecutive days. Statistical evaluation revealed relative standard deviations, at different values (Table 1 a \& b).

\begin{tabular}{|c|c|c|c|c|}
\hline $\begin{array}{c}\text { Nominal } \\
\begin{array}{c}\text { Concentration } \\
(\mathrm{ng} / \mathrm{mL})\end{array}\end{array}$ & 120.598 & 65.123 & 5.444 & 1.821 \\
\cline { 2 - 5 } & 121.3964 & 67.1349 & 5.3583 & 1.7372 \\
\hline Mean & 5.20889 & 4.69218 & 0.22599 & 0.19099 \\
\hline SD & 4.29 & 6.99 & 4.22 & 10.99 \\
\hline \%CV & 100.66 & 103.09 & 98.4395 .40 & 95.40 \\
\hline \%Nominal &
\end{tabular}

Table 1a: Back calculated concentrations of quality control samples-between batches

\begin{tabular}{|c|c|c|c|c|}
\hline $\begin{array}{c}\text { Nominal } \\
\text { Concentration } \\
(\mathrm{ng} / \mathrm{mL})\end{array}$ & 120.598 & 65.123 & 5.444 & 1.821 \\
\cline { 2 - 5 } & 121.3964 & 67.1349 & 5.3583 & 1.7372 \\
\hline Mean & 5.20889 & 4.69218 & 0.22599 & 0.19099 \\
\hline SD & 4.29 & 6.99 & 4.22 & 10.99 \\
\hline$\% \mathrm{CV}$ & 100.66 & 103.09 & 98.4395 .40 & 95.40 \\
\hline
\end{tabular}

Table 1b: Back calculated concentrations of quality control samples-within batches 
The absolute recovery of the ergocalciferol and IS were measured by comparison of peak area ratios of extracted samples of spiked matrices versus non-extracted standard solutions at three concentration levels. Recovery rates are in the range from 95.25 $-104.52 \%$. Satisfactory repeatability and precision was achieved with global SD values lower than $10 \%$ (Table 2).

\begin{tabular}{|c|c|c|c|}
\hline $\begin{array}{c}\text { Mean recovery of } \\
\text { analyte }\end{array}$ & 98.79 & $\begin{array}{l}\text { Mean Recov- } \\
\text { ery of Internal } \\
\text { standard }\end{array}$ & 100.27 \\
\hline Global CV & 3.33 & Global CV & 3.93 \\
\hline
\end{tabular}

\section{Stability}

Long term stock solution stability was evaluated during sample storage at $2-8^{\circ} \mathrm{C}$. Ergocalciferol and IS (D $)$ were found to be stable for 04 days 17 minutes and 04 days and 14 minutes respectively for analyte and IS. The \% stability found to be 100.93 for the analyte and 101.21 for the IS. Long term working solution stability for the analyte and IS were found to be stable for 03 days, 23 hours, 30 minutes and 03 days, 23 hours and 25 minutes respectively. At HQC level the stability of the analyte and IS found to be 100.44 and 101.08 and at LQC level 96.41 and 102.71 respectively. Stability was checked after 5 freeze thaw cycles and was found to be stable in human plasma after for 5 freeze-thaw cycles with percentage accuracy of 98.27, 103.38, for HQC and LQC respectively at -70 ${ }^{\circ} \mathrm{C}$ (Table 3 a \& b). Stability was also checked at $-20^{\circ} \mathrm{C}$ and ergocalciferol was found to be stable for 03 days, 14 hours, 35 minutes with percentage accuracy of 99.90 and $104.28 \%$ for HQC and LQC respectively. The representative chromatograms are shown in Figure 3 for blank plasma, $\mathrm{D}_{3}$ cholicalciferol and $\mathrm{D}_{2}$ ergocalciferol, plasma spiked with $\mathrm{D}_{2}$ and $\mathrm{D}_{3}$ at LLOQ, LQC, MQC and HQC. The chromatogram has a well resolved peak without any interference.

\begin{tabular}{|c|c|c|c|c|}
\hline \multicolumn{5}{|c|}{ Freeze Thaw Stability } \\
\hline \multirow{4}{*}{ S.No } & \multicolumn{2}{|c|}{ At 0 hour } & \multicolumn{2}{|c|}{ After 5 cycles } \\
\hline & HQC & LQC & HQC & LQC \\
\hline & \multicolumn{4}{|c|}{ Actual Concentration (ng/ml) } \\
\hline & 120.960 & 5.461 & 120.598 & 5.444 \\
\hline 1. & 119.880 & 5.991 & 122.770 & 5.818 \\
\hline 2. & 117.642 & 5.652 & 118.818 & 5.512 \\
\hline 3. & 114.121 & 6.017 & 120.839 & 5.641 \\
\hline 4. & 120.051 & 5.539 & 115.444 & 5.365 \\
\hline 5. & 119.264 & 6.031 & 117.530 & 5.906 \\
\hline 6. & 120.475 & 5.673 & 115.689 & 5.525 \\
\hline \multicolumn{3}{|c|}{ Mean } & 118.5150 & 5.6278 \\
\hline \multicolumn{3}{|c|}{ S.D \pm} & 2.89777 & 0.20337 \\
\hline \multicolumn{3}{|c|}{$\% \mathrm{CV}$} & 2.45 & 3.61 \\
\hline \multicolumn{3}{|c|}{$\%$ Nominal } & 98.27 & 103.38 \\
\hline
\end{tabular}

Table 3a: Freeze thaw stability

\begin{tabular}{|c|c|c|}
\hline S.No & \multicolumn{2}{|c|}{ Long term stock solution and working solution stability } \\
\hline 1. & $\begin{array}{r}\text { \% stability of the analyte after 4 days in } \\
\text { stock solutions }\end{array}$ & 100.93 \\
\hline 2. & $\begin{array}{r}\text { \% stability of Internal standard after } 4 \\
\text { days in stock solutions }\end{array}$ & 101.21 \\
\hline 3. & $\begin{array}{r}\text { Long term working solution stability at } \\
\text { HQC level }\end{array}$ & 100.44 \\
\hline 4. & $\begin{array}{r}\text { Long term working solution stability of } \\
\text { Internal standard at HQC level }\end{array}$ & 101.08 \\
\hline 5. & $\begin{array}{r}\text { Long term working solution stability of } \\
\text { analyte at LQC level }\end{array}$ & 96.41 \\
\hline 6. & $\begin{array}{r}\text { Long term working solution stability of } \\
\text { Internal standard at LQC level }\end{array}$ & 102.71 \\
\hline
\end{tabular}

Table 3b: Long term stock solution and working solution stability

\section{Discussion}

Determination of vitamin $\mathrm{D}$ is required not only to detect for its deficiency, but also increasingly to adjust therapeutic targets 
and monitor safety and efficacy of treatment. In addition to the skeletal effects, vitamin D may have a role in relation to diabetes, cancer and cardiovascular diseases [24-26]. Increasing awareness of the clinical importance of vitamin D has resulted in diagnostic laboratories receiving requests for the assay. This has led to a need, to more highly automated testing equipments and to use mass spectra. Previously the major challenge with vitamin D analysis has been the lack of standardization and the wide variation between analytical methods due to absence of reference standards [27]. The situation is further complicated by the discovery of C-3 epimers of vitamin $\mathrm{D}_{2}$ and $\mathrm{D}_{3}$ particularly in infants that might interfere with the accurate measurement and interpretation of vitamin D status in infants [28].

A.

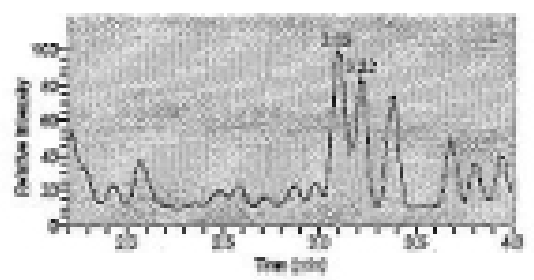

B.

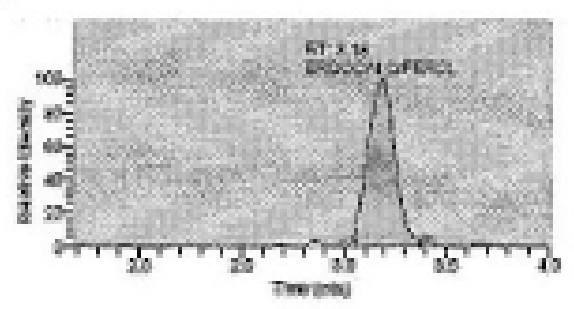

c.

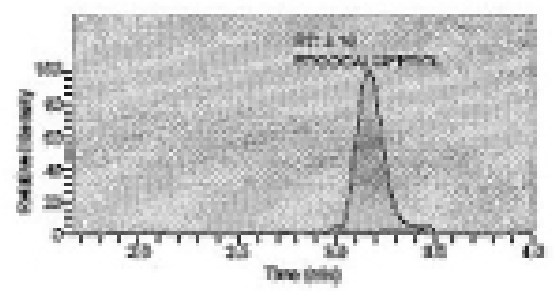

D.

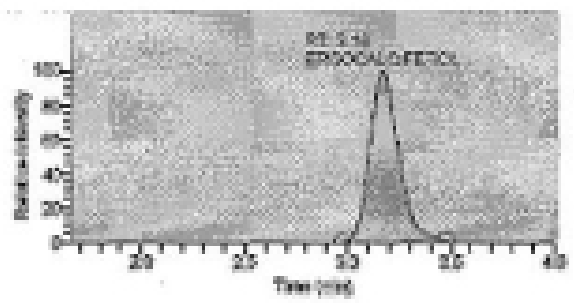

E.

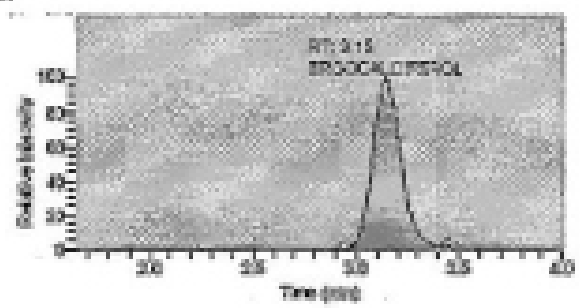

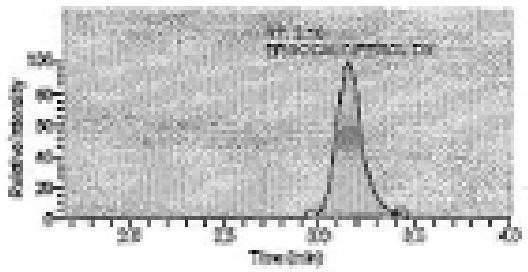
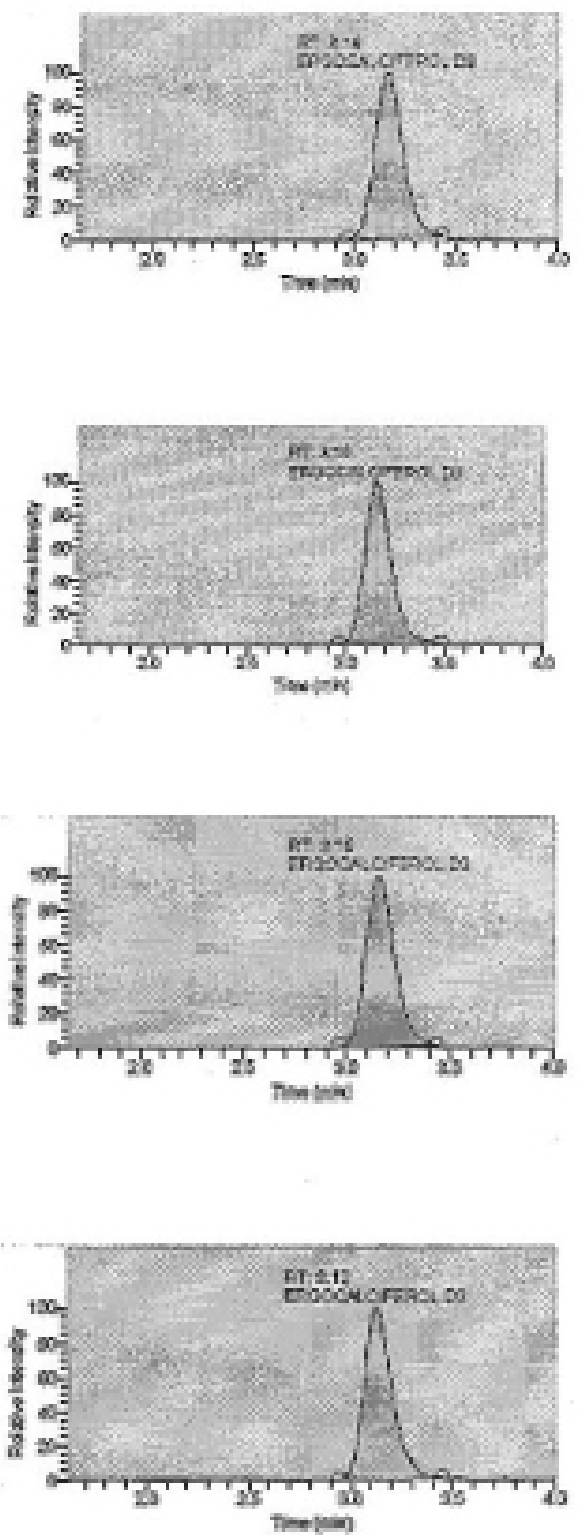

Figure 3: Representative chromatograms of blank + internal standard, LLOQC, LQC, MQC and HQC. (A). Representative ion chromatogram for blank + Internal standard (B). Representative ion chromatogram for LLOQ QC (C). Representative ion chromatogram for LQC (D). Representative ion chromatogram for MQC (E). Representative ion chromatogram for HQC 
Erogcalciferol was determined by using a fully validated, modified high-performance liquid chromatography (HPLC) method [2]. In this method, protein is precipitated, and through selective solid phase extraction, interfering components are removed and the analytes are concentrated. A stable vitamin $\mathrm{D}_{3}$ is used as an internal standard in order to allow for accurate quantification. The chromatographic separation takes approximately $12 \mathrm{~min}$. The present method has a run time of 4 minutes which is fastest method compared to other methods published recently [29]. Due to low ionization efficiency analysis of vitamin D metabolites is difficult. But by using APCI increases its sensitivity. PTAD is one of the strongest dienophiles for derivatizing vitamin D forms [29] which also improves the sensitivity of analyte 10-100 fold. LLE extraction is able to separate components in the solvent mixture which are present in low concentration and its varying solubility. Stage wise phase contact enhances the efficiency of the extraction system.

The developed method is very specific to quantify the ergocalciferol because of a liquid-liquid extraction procedure and easy to handle LC-MS/MS system. We used vitamin $\mathrm{D}_{3}$ as an internal standard. Regarding stability, the results were shown that ergocalciferol was found stable for 3 days at $-70{ }^{\circ} \mathrm{C}$ (98-103\%) and after five cycles of freeze thaw. Our method is very specific to quantify ergocalciferol in human plasma [31]. Retention time is faster i.e. 3.5 minutes when compared to the recently published method of $\mathrm{D}_{2}$ ergocalciferol [29] has the RT at 5.0 minutes. Similar detection of vitamin D and its metabolites in blood serum is analysed using HPLC by Samanidou et al. [32] and LC-MS/MS has been used for the determination of hydroxyl metabolites of vitamin D [33,34]. Similar studies were done using derivatization method in liver, gastrocnemius muscle, and epididymal fat homogenates [35] and in human serum [36]. High specificity and sensitivity is achieved using PTAD derivatization followed by LC-MS/MS in vitamin $\mathrm{D}$ metabolites $[1,3,4,9,10]$. The LC-MS/MS method is a completely validated method and is specific and selective for measuring plasma levels of vitamin $\mathrm{D}_{2}$ at nanogram concentrations. In addition this method proves that it is a reproducible method and has a short analysis time. Since the level of ergocalciferol in human plasma is considered as important parameter in determining human body health status, the present method helps to determine ergocalciferol levels in human plasma but also increasingly to adjust therapeutic targets and monitor safety and efficacy of treatment. This method helps to analyze, and research on the significance of vitamin $\mathrm{D}_{2}$ plasma concentrations in connection with disease states and supplementation compliance.

\section{Conclusion}

A method using LC-MS/MS with APCI mode was developed and validated to determine ergocalciferol in human plasma. In this method evaluation of extraction efficiency showed that protein precipitation followed by liquid-liquid extraction given the highest extraction of ergocalciferol with high reproducibility by using acetonitrile and methanol. In this method, LOQ is determined as $0.495 \mathrm{ng} / \mathrm{mL}$. The best fit of individual standard curve was achieved using a first-order linear regression and showed good linearity within the tested ranges $\left(\mathrm{R}^{2} \geq 0.99\right)$. PTAD reagent showed exceptional ability to derivatize ergocalciferol and increased the sensitivity to have good reproducibility. Further, this method can be successfully applied to determine the levels of ergocalciferol in plasma samples from volunteers participating in clinical study. This method is validated as per FDA guidance for industry and assured standardized validation process. LC-MS/MS assay can be used for quantifying ergocalciferol native form from external consumption and study the metabolism of each individuals enrolled in the clinical trials. This method can be applied for pharmacokinetic studies where lot of samples needs analysis for ergocalciferol quantification.

\section{Acknowledgement}

We acknowledge the personnel who helped throughout the project.

\section{Competing interests}

The authors declare no conflict of interest.

\section{References}

1. Viljakainen HT, Korhonen T, Hytinantti T, Laitinen EK, Amdersson S, et al. (2011) Maternal vitamin D status affects bone growth in early childhood--a prospective cohort study. Osteoporosis Int 22: 883-91.

2. Ousteata M, Ousteata FM (2011) Overview of extraction methods for analysis of vitamin D and its metabolites in biological samples. Bioanalysis 17: 1987-2002.

3. Trang HM, Cole DE, Rubin, Pierratos A, Siu S, et al. (1998) Evidence that vitamin $\mathrm{D}_{3}$ increases serum 25 -hydroxyvitamin D more efficiently than does vitamin $\mathrm{D}_{2}$. Am J Clin Nutri 68: 854-8.

4. Houghton LA, Vieth R (2006) The case against ergocalciferol (vitamin $\mathrm{D}_{2}$ ) as a vitamin supplement. Am J Clin Nutr 84: 694-7.

5. Liebisch G, Matysik S (2015) Accurate and reliable quantification of 25-hydroxyvitamin D species by liquid chromatography high-resolution tandem mass spectrometry. J Lipid Res 56: 1234-9.

6. Marwaha RK, Tandon N, Reddy D, Aggarwal R, Singh R, et al. (2005) Vitamin D and bone mineral density status of healthy schoolchildren in northern India. Am J clin. Nutr. 82: 477-82.

7. Van den Ouweland JMW, Beijers AM, Demacker PNM, Van Daal H (2010) Measurement of 25-OH-vitamin D in human serum using liquid chromatography tandem-mass spectrometry with comparison to radioimmunoassay and automated immunoassay. J Chrom B 878: 1163-8.

8. Brunetto MR, Obando MA, Gallignani M, Alarcón OM, Nieto E, et al. (2004) HPLC determination of Vitamin D3 and its metabolite in human plasma with on-line sample cleanup. Talanta 64: 1364-70.

9. Holick MF (2006) Resurrection of vitamin D deficiency and rickets. J Clin Invest 116: 2062-72.

10. Oymoller L, Jensen SK (2011) Vitamin D analysis in plasma by high performance liquid chromatography (HPLC) with C (30) reversed phase column and UV detection--easy and acetonitrile-free. J Chrom A 1218: 1835-41. 
11. Optimising the derivatisation of vitamin $\mathrm{D}$ metabolites within clinical research using Amplifex ${ }^{\mathrm{Tm}}$ Diene. Reagent.

12. Hedman CJ, Wiebe DA, Dey S, Plath J, Kemnitz JW, et al. (2014) Development of a sensitive LC/MS/MS method for vitamin D metabolites: 1,25 Dihydroxyvitamin D2\&3 measurement using a novel derivatization agent. Chromatogr B Analyt Technol Biomed Life Sci 15: 953-4.

13. Zusammenfassung (2013) Quantitative analysis of vitamin D3, vitamin D2, and metabolites there of US 8617898 B2.

14. Curtis JH, Donald AW, Subhakar D, Josh P, Joseph WK, Toni EZ (2014) Development of a sensitive LC-MS/MS method for vitamin D metabolites: 1,25 Dihydroxyvitamin D2\&3 measurement using a novel derivatization agent. J Chromatography B. 953-954: 62-67.

15. Tristan EL, Bruce RC, Emily EH, Connie MW, Mario GF, et al. (2013) Quantification of vitamin D and 25-hydroxyvitamin D in soft tissues by liquid chromatography-tandem mass spectrometry. J Chromatogr B Analyt Technol Biomed Life Sci 932: 6-11.

16. Wan D, Yang J, Barnych B, Hwang SH, Lee KS, et al. (2017) A new sensitive LC-MS/MS analysis of vitamin D metabolites using a derivatization reagent, 2-nitrosopyridine. J Lipid Res 58: 798-808.

17. Jenny P Dai, Allan Xu, Eric J Battaglioli, Bruce A Stanley, Robin T Wilson (2015) Sensitive and cost-effective LC-MS/MS method for determination of 1a,25Dihydroxyvitamin D3 in human plasma 13.

18. Burild A, Frandsen HL, Jakobsen J (2014) Simultaneous quantification of vitamin D3, 25-hydroxyvitamin D3 and 24,25-dihydroxyvitamin D3 in human serum by LC-MS/MS. Scandinavian Journal of Clinical and Laboratory Investigation 74: 418-23.

19. Xuejun Peng,Rong Yi, Amara Pinnawala,Sarah Ostonal,Eliot Chung,Grace van der Gugten,Bernard Nutley,David Gray (2009) Bioanalytical quantitation method for analysis of 1,25-Dihydroxyvitamin D3 (DHVD3) in human plasma using Amplifex ${ }^{\text {Tm }}$ Reagent Kit on SCIEX Triple Quad $^{\text {Ts }} 5500$ System.

20. Contractor P, Gandhi A, Solanki G, Shah PA, Shrivasta PS (2017) Determination of ergocalciferol in human plasma after Diels-Alder derivatization by LC-MS/ MS and its application to a bioequivalence study. J Pharm Anal.

21. Satoh M, Ishige T, Ogawa S, Nishimura M, Matsushita K, et al. (2016) Development and validation of the simultaneous measurement of four vitamin D metabolites in serum by LC-MS/MS for clinical laboratory applications. Anal Bioanal Chem 408: 7617-7.

22. Jenkinson C, Taylor AE, Hassan-Smith ZK, Adams JS, Stewart PM, et al. (2016) High throughput LC-MS/MS method for the simultaneous analysis of multiple vitamin D analytes in serum. J Chromatogr B Analyt. Technol Biomed Life Sci 1014: 56-63.

23. FDA Guidance for Industry, Bioanalytical Method Validation, US Department of Health and Human Services Food and Drug Administration, May 2001.

24. Rosen CJ (2011) Vitamin D Insufficiency N Engl. J Med 364: 248-54.

25. Hanley DA, Cranney A, Jones G, Whiting SJ, Leslie WD, et al. (2010) Vitamin D in adult health and disease: a review and guideline statement from Osteoporosis. Canada Can Med Assoc J 182: E610-8.

26. Manson JE, Mayne ST, Clinton SK (2011) Vitamin D and Prevention of cancer-ready for prime time? N Engl.J.Med.364: 1385-1387.

27. Phinney KW (2008) Development of a standard reference material for vitamin D in serum1,2,3,4. Am J Clin Nutr 88: 511 - 12.

28. Singh RJ, Taylor RL, Reddy GS, Grebe SKG (2006) C-3 epimers can account for a significant proportion of total circulating 25-hydroxyvitamin D in infants, complicating accurate measurement and interpretation of vitamin D status. J Clin Endocrinol Metab 91: 3055-61.

29. Higashi T, Shibayama Y, Fuji M, Shimada K (2008) Liquid chromatography-tandem mass spectrometric method for the determination of salivary 25-hydroxyvitamin D3: a noninvasive tool for the assessment of vitamin D status. Anal Bioanal Chem 391: 229-38.

30. Liebisch, Silke Matysik (2015) Accurate and reliable quantification of 25-hydroxyvitamin D species by liquid chromatography high-resolution tandem mass spectrometry. J Lipid Res 56: 1234-8.

31. Syed NA, Ahmed Yusuf, Muhammad M (2012) Simultaneous quantification of vitamin D-2, Vitamin D-3, and their 25- Hydroxy metabolites in human plasma by high performance liquid chromatography. J Bioequiv Availab S14.

32. Samanidou VF, Sofia C, Vardali, Loannis NP (2013) Development and validation of a high pressure liquid chromatographic method for the simultaneous determination of four vitamin D metabolites in blood serum. J App Biopharmaceutics Pharmacokinetics 1: 72-9.

33. Yu Y, Pan Y, Cao Y, Wu J, Lai G (2009) Identification and structural elucidation of vitamin D3 metabolites in human urine using LC-MS/MS. Chromatographia 69: 103-9.

34. Adamec, J, Jannasch, A, Huang J, Hohman E, Fleet JC, Munro Peacock, Mario G. Ferruzzi, Berdine Martin, Connie MW (2011) Development and optimization of an LC-MS/MS-based method for simultaneous quantification of vitamin D2, vitamin D3, 25- hydroxyvitamin D2 and 25-hydroxyvitamin D3. J Sep Sci 1: 11-20.

35. Pauline Le Faouder, Vincent Baillif, Ian Spreadbury, Jean-Paul Motta, Perrine Rousset, et al. (2013) LC-MS/MS method for rapid and concomitant quantification of pro-inflammatory and pro-resolving polyunsaturated fatty acid metabolites. Chromatogr. B Analyt. Technol. Biomed Life Sci 932: 123-33.

36. Miriam JM, Caroline SS, Lammert F, Dietrich AV (2016) Chemotyping the distribution of vitamin D metabolites in human serum. Nature Sci Rep 6: 21080.

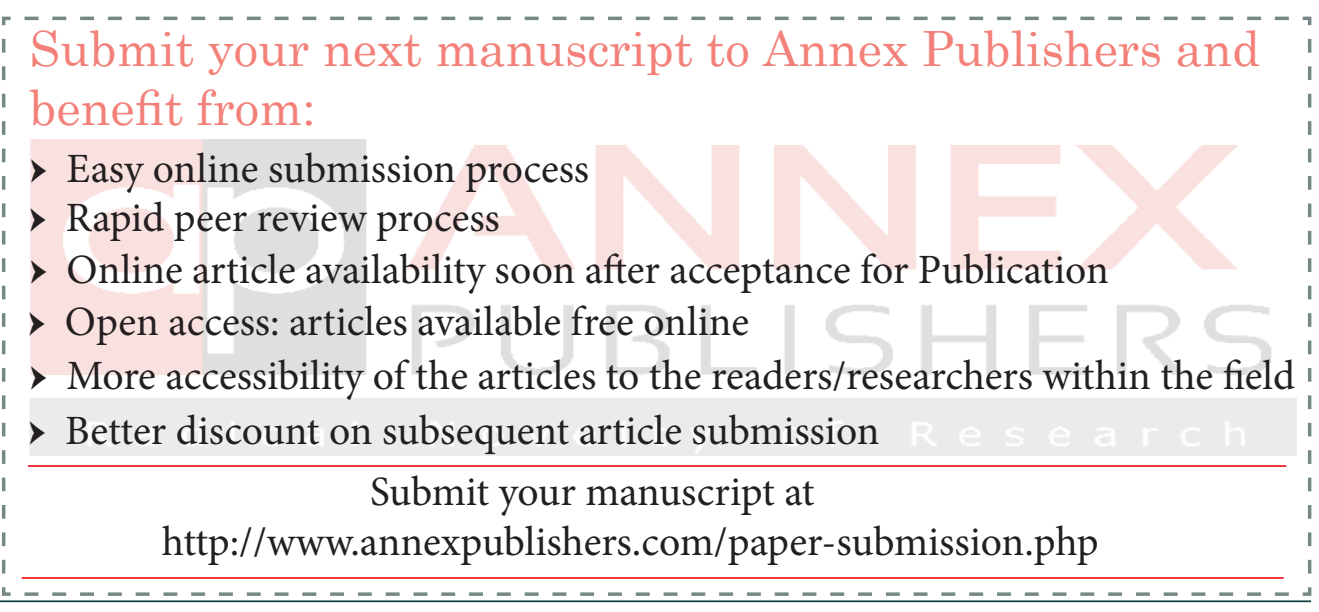

